\title{
Safety of Bariatric Surgery During the Opening Phase After the First Wave of the COVID-19 Pandemic: Experience at an Academic Center
}

\author{
Fernando Crovari ${ }^{1} \cdot$ Martin Inzunza $^{1} \cdot$ María J. Irarrázaval ${ }^{1} \cdot$ Cecilia Romero $^{1} \cdot$ Pablo Achurra $^{1} \cdot$ Nicolás Quezada $^{1}$. \\ Mauricio Gabrielli ${ }^{1} \cdot$ Rodrigo Muñoz $^{1,2}$
}

Received: 16 July 2021 / Revised: 26 August 2021 / Accepted: 29 August 2021 / Published online: 4 September 2021

(c) The Author(s), under exclusive licence to Springer Science+Business Media, LLC, part of Springer Nature 2021

\begin{abstract}
Introduction Increased morbimortality in patients with COVID-19 infection who had undergone surgery has raised concerns about bariatric surgery safety during the current COVID-19 pandemic. Currently, there is scarce literature on safety outcomes after bariatric surgery during the COVID-19 pandemic.

Objectives To determine the risk of symptomatic COVID-19 infection and associated complications during the first 30 days after bariatric surgery.

Materials and Methods Prospective observational cohort study including all patients who consecutively underwent primary bariatric surgery between August and December 2020.

Results A total of 189 patients were included. Median age and BMI were $36(17-70)$ years and $38(35-41) \mathrm{kg} / \mathrm{m}^{2}$, respectively. Forty percent of patients were women $(n=76), 59.3 \%(n=112)$ underwent sleeve gastrectomy (SG), and $40.7 \%$ $(n=77)$ underwent Roux-en-Y gastric bypass (RYGB). All surgeries were performed laparoscopically. The median length of postoperative stay was $2(0-5)$ days. Postoperative COVID-19 infection was detected in two patients (1.1\%): one patient was readmitted without the need of intermediate or ICU care, and the other was managed as an outpatient. Major complications occurred in three patients (1.6\%); none of them was COVID-19 related. Two patients required an unplanned reoperation. No patient required intermediate or ICU care, no severe COVID-19 complications were observed, and no mortality was reported. Conclusion Bariatric surgery can be safely performed during the ongoing pandemic, albeit a low risk of COVID-19 symptomatic infection. Rigorous perioperative COVID-19 institutional protocols are required to perform bariatric surgery safely during the current pandemic.
\end{abstract}

Keywords COVID-19 · Bariatric surgery · Roux-en-Y gastric bypass · Sleeve gastrectomy

Key Points 1 . The risk of symptomatic COVID-19 infection after primary bariatric surgery is low.

2. In 189 patients, only two $(1.1 \%)$ had symptomatic COVID-19 infection in the first 30 days after surgery.

3. No severe COVID-19-associated complications were reported.

4. Major complications occurred in $1.6 \%$ of patients. No intermediate or ICU care was required in any of these patients.

Rodrigo Muñoz

rmunozc@med.puc.cl

1 Department of Digestive Surgery, School of Medicine, Pontificia Universidad Católica de Chile, Santiago, Chile

2 Santiago, Chile

\section{Introduction}

The severe acute respiratory syndrome (SARS) caused by the coronavirus 2 (SARS-CoV-2) infection has globally spread since early 2020 , causing an unprecedented pandemic with enormous social, economic, and health consequences. At the beginning of the pandemic declared by the World Health Organization (WHO) on March 11, 2020, most of the elective surgical procedures were canceled. In Chile, phase 4 of the pandemic was declared on March 15, 2020, and several measures were implemented to limit the spread of the virus and to optimize human and infrastructural hospital resources to face the growing pandemic. In addition, public and private health institutions postponed most elective surgeries, prioritizing patients with surgical emergencies or oncological diseases. As the first wave of the pandemic hit 
our country, surgical treatment of patients with obesity was canceled until surgeries were opened again in August 2020.

After the beginning of the pandemic, evidence showed that perioperative COVID-19 infection in patients undergoing unpostponable surgeries significantly increased their risk of severe complications and mortality [1]. Moreover, early reports also showed unexpected high mortality due to COVID-19-associated complications in a set of patients undergoing general surgery procedures and bariatric surgery early at the beginning of the pandemic [2,3]. Our group reported our perioperative results in a cohort of 701 consecutive patients who underwent unpostponable oncological and emergency surgeries and found that patients with perioperative COVID-19 infection had a higher 30-day rate of overall complications, COVID-19-associated complications, and mortality [4]. We observed a 30-day mortality rate of $12.8 \%$ in patients with COVID-19 infection and $1.4 \%$ in patients without infection, which is lower than the $23.8 \%$ mortality reported in the most extensive study published today [1]. Together, the increased risk of surgical complications due to perioperative COVID-19 infection in patients with obesity - one of the most important risk factors for a severe COVID-19 infection, as shown by higher hospitalization rates, intensive care unit admission, invasive mechanical ventilation, and mortality [5-9]—and potential limited access to critical care units during periods of high viral circulating levels have raised concerns about the safety of bariatric surgery and have led to suspension of surgical treatment of obesity. The implications of these widespread suspensions of the most effective treatment for weight loss in patients with a higher risk of severe COVID-19 complications are still unknown. Surgical scientific societies have developed guidelines to safely resume bariatric surgery which along with institutional strict perioperative COVID-19 protocols allowed the start of surgical procedures [10-12].

Over the last year, few studies have evaluated the safety of bariatric surgery during the opening phases between COVID-19 pandemic waves. The results are encouraging as they show a low risk of postoperative COVID-19 infection, and therefore a low risk of COVID-19 severe complications [13-15]. Moreover, preliminary evidence also suggests that bariatric surgery could protect against severe forms of COVID-19 infection in patients with prior bariatric surgery [16].

In this study, we sought to evaluate the safety of bariatric surgery by determining the risk of symptomatic COVID19 infection and associated complications during the first 30 days after surgery in a group of consecutive patients who underwent primary bariatric surgery during the opening phase for surgery between the first and second waves of the COVID-19 pandemic. As a secondary outcome, we also determined the risk of severe complications and intermediate/intensive care unit admission requirements.

\section{Methods}

\section{Study Design}

This prospective observational cohort study was conducted at the Hospital Clínico Pontificia Universidad Católica, Chile, between August and December 2020. Our institutional ethics committee reviewed and approved the study.

\section{Patients}

We included all patients $\geq 15$ years old who consecutively underwent a primary laparoscopic Roux-en-Y gastric bypass (RYGB) or sleeve gastrectomy (SG) after a multidisciplinary evaluation at our Center for Obesity Treatment, Department of Digestive Surgery, Pontificia Universidad Católica, Chile.

\section{Surgical Technique}

SG is performed by resecting 75 to $80 \%$ of the stomach alongside a 50-French bougie beginning 5 to $6 \mathrm{~cm}$ from the pylorus and ending at the His angle. A running suture along the stapler line is routinely performed using an absorbable suture. RYGB is performed using five ports, constructing a 10-15-cc gastric pouch, with a complete transection of the stomach leaving a $150-\mathrm{cm}$ antecolic, antegastric alimentary limb and a 50-cm biliary limb. Mesenteric defects are routinely closed using a nonabsorbable suture. A running suture over the staple line of the gastric pouch is routinely performed using an absorbable suture. The gastrojejunostomy is performed with a handsewn double-layer technique and calibrated over a $34-\mathrm{F}$ bougie.

\section{Clinical Variables and Follow-up}

The following variables were registered for analysis: age, gender, comorbidities, body mass index (BMI), prior symptomatic SARS-CoV-2 infection, type of surgery (RYGB and SG), hospital length of stay, postoperative symptomatic SARS-CoV-2 infection, morbidity, and mortality. Complications were registered and graded using the Clavien-Dindo classification [17, 18]. Major complications were defined as Clavien-Dindo (CD) score $\geq$ III. Postoperative follow-up was carried out until 30 days after surgery and included outpatient visits, telemedicine, and telephone calls [19]. Reoperations and readmissions were also considered within 30 days of surgery. 


\section{Primary and Secondary Outcomes}

The primary outcome was symptomatic COVID-19 infection and associated complications (e.g., pneumonia, respiratory distress, need for noninvasive mechanical ventilation (IMV), intermediate or ICU care, mortality) during the first 30 days after surgery. Secondary outcomes included complications $\mathrm{CD} \geq \mathrm{III}$, readmissions, reoperation, and need of intermediate or ICU care.

\section{Institutional COVID-19 Protocol for Elective Surgery}

COVID-19 status was determined based on the results of viral SARS-CoV-2 RNA detection by quantitative RT-PCR up to $48 \mathrm{~h}$ before surgery through nasopharyngeal swabs according to the standardized technique. Postoperative COVID-19 infection was defined in all patients with SARSCoV-2 symptoms that tested positive during the 30-day follow-up. Institutional protocols were adopted to care for patients from arrival to the preoperative, ward, and postoperative recovery [4]. During the 30-day follow-up, PCR tests were indicated if the patient developed fever or respiratory symptoms.

\section{Statistical Analysis}

Statistical analysis was performed with SPSS 25 (IBM Corp., Armonk, NY). All data are expressed as mean and standard deviation or median and range.

\section{Results}

During the opening phase for bariatric surgery between August and December 2020 of the ongoing COVID-19 pandemic in Chile, 189 patients underwent a primary laparoscopic bariatric surgery, 112 patients $(59.3 \%)$ underwent SG, and 77 patients $(40.7 \%)$ underwent RYGB. The median age of patients was $36(17-70)$ years, and the median BMI was $38(35-41) \mathrm{kg} / \mathrm{m}^{2}$. Table 1 summarizes baseline patient characteristics. Before surgery, six $(3.2 \%)$ patients had COVID-19 infection, two patients were asymptomatic, and four patients developed a mild symptomatic infection.

During the first 30 days after discharge, symptomatic SARS-CoV-2 infection was detected in two patients (1.1\%) who reported upper respiratory tract infection symptoms. One 49-year-old woman developed mild respiratory symptoms 18 days after SG and was readmitted for observation. At her readmission, no pneumonia was detected on the chest CT and did not require intermediate or ICU care. After 2 days, the patient was discharged. Another 36-yearold woman developed symptoms 14 days after SG and was
Table 1 Baseline patient characteristics

\begin{tabular}{llll}
\hline & Total $(n=189)$ & RYGB $(n=77)$ & SG $(n=112)$ \\
\hline $\begin{array}{l}\text { Age; years } \\
\text { Sex }\end{array}$ & $37.1 \pm 9.8$ & $39.7 \pm 9.8$ & $35.3 \pm 9.4$ \\
$\quad$ Male & $44(23.3 \%)$ & $23(29.9 \%)$ & $21(18.8 \%)$ \\
$\quad$ Female & $145(76.7 \%)$ & $54(70.1 \%)$ & $91(81.2 \%)$ \\
ASA classification & & & \\
$\quad$ I & $21(11.1 \%)$ & $9(11.7 \%)$ & $12(10.7 \%)$ \\
II & $167(88.4 \%)$ & $68(88.3 \%)$ & $99(88.4 \%)$ \\
III & $1(0.5 \%)$ & $0(0 \%)$ & $1(0.9 \%)$ \\
Preoperative BMI & & & \\
$\quad 30-34.9$ & $36(19 \%)$ & $7(9.1 \%)$ & $29(25.9 \%)$ \\
$\quad 35-39.9$ & $89(47.1 \%)$ & $26(33.8 \%)$ & $63(56.3 \%)$ \\
$\geq 40$ & $64(33.9 \%)$ & $44(57.1 \%)$ & $20(17.9 \%)$ \\
Past medical his- & $6(3.4 \%)$ & $2(2.6 \%)$ & $4(3.6 \%)$ \\
$\quad$ tory of COVID- & & & \\
19 & & & \\
\hline
\end{tabular}

$R Y G B$, Roux-en-Y gastric bypass; $S G$, sleeve gastrectomy; $A S A$, American Society of Anesthesiologists; $B M I$, body mass index.

${ }^{\mathrm{a}}$ Mean \pm standard deviation.

managed as an outpatient. Therefore, no severe COVID19-related complications were observed in this group of patients. Characteristics of patients with perioperative SARS-CoV-2 infection are described in Table 2.

After surgery, the median length of postoperative stay was 2 days (0-5) (Table 3). No conversion to open surgery was reported. Early complications occurred in 12 patients (6.3\%), of which three (1.6\%) were major complications. Two patients required reoperation. One RYGB-operated patient required early reoperation before discharge due to a gastrojejunal anastomosis (GJA) stricture. One SGoperated patient was readmitted 7 days after discharge due to abdominal pain and a slight elevation in white blood cell count. An abdominal computed tomography revealed no signs of leaks and laminar fluid collection. The attending surgeon decided to undergo an exploratory laparoscopy which revealed blood clots along the stapler line without intraoperative evidence of leakage. The patient was discharged after 6 days; no other complications were reported. Importantly, none of those abovementioned patients required intermediate or ICU care (Table 4). No mortality was reported.

During the first 30 days after surgery, hospital readmission occurred in seven $(3.7 \%)$ patients, four after RYGB, and three after SG. The causes of hospital readmission were dehydration $(n=1)$, symptomatic COVID-19 infection $(n=1)$, bowel obstruction $(n=2)$, GJA stricture $(n=1)$ that required an endoscopic balloon dilation, and abdominal pain $(n=2)$. Table 5 summarizes the causes of hospital readmission. 


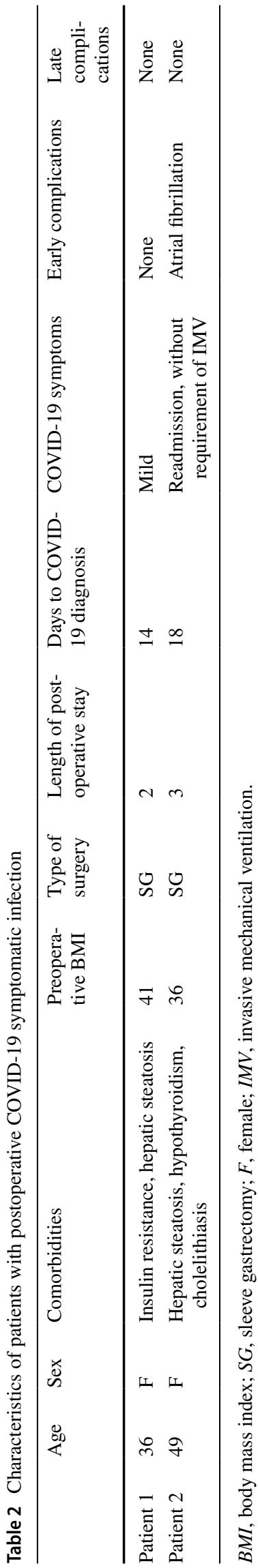

Table 3 Perioperative variables

\begin{tabular}{llll}
\hline & Total $(n=189)$ & RYGB $(n=77)$ & SG $(n=112)$ \\
\hline Type of surgery & & & \\
RYGB & $77(40.7 \%)$ & $77(100 \%)$ & $0(0 \%)$ \\
SG & $112(59.3 \%)$ & $0(0 \%)$ & $112(100 \%)$ \\
Additional surgery & $17(9 \%)$ & $8(10.4 \%)$ & $9(8 \%)$ \\
Cholecystectomy & $14(7.4 \%)$ & $6(7.8 \%)$ & $8(7.1 \%)$ \\
Hernioplasty & $2(1.1 \%)$ & $1(1.3 \%)$ & $1(0.9 \%)$ \\
Other & $1(0.5 \%)$ & $1(1.3 \%)$ & $0(0 \%)$ \\
$\begin{array}{c}\text { Length of postop- } \\
\text { erative stay; days }\end{array}$ & $1.9 \pm 0.7$ & $2.1 \pm 0.7$ & $1.8 \pm 0.6$ \\
\hline
\end{tabular}

$R Y G B$, Roux-en-Y gastric bypass; $S G$, sleeve gastrectomy.

${ }^{\mathrm{a}}$ Mean \pm standard deviation.

Table 4 Early complications

\begin{tabular}{llll}
\hline & Total $(\boldsymbol{n}=189)$ & RYGB $(\boldsymbol{n}=77)$ & SG $(\boldsymbol{n}=112)$ \\
\hline $\begin{array}{l}\text { Postoperative } \\
\text { COVID-19 } \\
\text { infection }\end{array}$ & $2(1.1 \%)$ & $0(0 \%)$ & $2(1.8 \%)$ \\
$\begin{array}{l}\text { Clavien-Dindo } \\
\text { I-II }\end{array}$ & $9(4.2 \%)$ & $5(6.4 \%)$ & $4(3.6 \%)$ \\
$\geq$ III & $3(1.6 \%)$ & $2(2.6 \%)$ & $1(0.9 \%)$ \\
Mortality & $0(0 \%)$ & $0(0 \%)$ & $0(0 \%)$ \\
Reoperation & $2(1.1 \%)$ & $1(1.3 \%)$ & $1(0.9 \%)$ \\
Readmission & $7(3.7 \%)$ & $4(5.2 \%)$ & $3(2.7 \%)$ \\
\hline
\end{tabular}

$R Y G B$, Roux-en-Y gastric bypass; $S G$, sleeve gastrectomy.

\section{Discussion}

Concerns about the potential risk of postoperative COVID19 infection, along with limited access to intermediate or ICU care, have raised concerns regarding the safety of bariatric surgery during the ongoing pandemic. Here, we report a $1.1 \%$ risk of symptomatic COVID-19 infection and no associated severe complications. Severe complications were observed in three patients (1.6\%), two of which required reoperation. No intermediate or ICU care was necessary. Together, these results indicate that bariatric surgery can be safely performed during the ongoing pandemic, albeit a minor risk for symptomatic COVID-19 infection during the first 30 days after surgery.

Perioperative COVID-19 infection has negatively impacted the safety of patients undergoing surgery, increasing complications and mortality rates $[1,4]$. Currently, only a few studies have reported data on the risk of perioperative COVID-19 infection and safety outcomes in patients undergoing bariatric surgery during the ongoing pandemic $[13-15,20]$. One study reported 87 consecutive 
Table 5 Characteristics of readmitted patients

\begin{tabular}{|c|c|c|c|c|c|c|c|c|}
\hline & Age & Sex & $\begin{array}{l}\text { Preop- } \\
\text { erative } \\
\text { BMI }\end{array}$ & Type of surgery & $\begin{array}{l}\text { Length of } \\
\text { postoperative } \\
\text { stay }\end{array}$ & Cause of readmission & $\begin{array}{l}\text { Days to } \\
\text { readmis- } \\
\text { sion }\end{array}$ & Reoperation/intervention \\
\hline Patient 1 & 21 & F & 40 & SG & 3 & Dehydration & 24 & No \\
\hline Patient 2 & 49 & $\mathrm{~F}$ & 36 & SG & 3 & COVID symptomatic infection & 18 & No \\
\hline Patient 3 & 26 & M & 44 & RYGB & 2 & Abdominal pain & 7 & No \\
\hline Patient 4 & 54 & $\mathrm{~F}$ & 39 & RYGB & 2 & Ileus & 10 & No \\
\hline Patient 5 & 33 & M & 38 & RYGB & 2 & Ileus & 9 & No \\
\hline Patient 6 & 43 & M & 39 & RYGB & 1 & GJA stricture & 25 & Yes, balloon dilatation \\
\hline Patient 7 & 48 & $\mathrm{~F}$ & 36 & SG & 3 & Abdominal pain & 7 & Yes, exploratory laparoscopy \\
\hline
\end{tabular}

$B M I$, body mass index; $R Y G B$, Roux-en-Y gastric bypass; $S G$, sleeve gastrectomy; $F$, female; $M$, male; $G J A$, gastrojejunal anastomosis.

patients who underwent SG during the opening phase between the first and second COVID-19 waves [14]. Postoperative symptomatic COVID-19 infection was reported in $6.3 \%$ of patients, with one patient having COVID-associated pneumonia during the first day after surgery with a negative preoperative PCR test. Another study involving 300 consecutive patients reported no symptomatic COVID-19 infection during the first 30 days after surgery [15]. Another study that included 178 patients reported $3.4 \%$ of patients who tested positive for COVID-19 without COVID-19-related readmission or mortality [20]. Finally, in the most extensive study that included 2001 patients from different countries, symptomatic COVID-19 infection was reported in $0.5 \%(n=10)$ patients during the first 30 days after surgery [13]. No mortality nor pulmonary complication in this group of patients was reported [13]. Our results are similar, with a low risk of postoperative symptomatic COVID-19 infection or associated severe complications. Moreover, we also reported a low rate of 30-day severe complications of $1.6 \%$. Finally, no intermediate or ICU care was required in the management of patients with complications in this study. The safety of bariatric surgery and the low risk of COVID-19 infection shown in our and previous studies are encouraging. It is possible that we will continue to face the challenges imposed by the current pandemic in the foreseeing future; therefore, safe access to the most effective treatment in patients with obesity needs to continue. Of importance is the observation of the low rate of perioperative COVID-19 infection and severe complications in patients undergoing bariatric surgery compared with the high risk of complications shown in the previous where $40-50 \%$ of patients developed pulmonary complications and had mortality rates between 20 and 25\% [2, 21-29]. However, these differences may be explained because these studies differ in the time of the pandemic (early vs. late), the types of surgical procedures performed, patients' characteristics, and the existence of perioperative COVID-19 protocols.
Despite these dissimilarities, the low rates of perioperative COVID-19 infection and related complications in our and other studies indicate that bariatric surgery can be safely performed during opening periods of the ongoing pandemic.

Another concern is the risk of severe complications that can require intermediate or ICU beds, which are limited in most institutions during high circulating periods of the SARS-CoV-19 virus. Bariatric surgery has shown to be a safe intervention, with a low risk of severe complications and mortality. In this study, overall complications and major complications were $6.3 \%$ and $1.6 \%$, respectively. No mortality was reported, and no need for an intermediate or ICU bed was required. These results are in accordance with previous studies that report overall complications rates between 1 and $6.8 \%$, major complications between 1 and $2.4 \%$, and mortality rates between 0 and $0.5 \%[13-15,20]$. Combined, these results are in line with pre-pandemic reported outcomes, indicating the safety of bariatric surgery with morbidity and mortality rates of $5 \%$ or less and $0.1 \%$, respectively [30].

In addition to the safety of bariatric surgery and its wellknown health benefits, new evidence indicates that it can also decrease the risk of severe forms of COVID-19 infection. A retrospective matched cohort studied the rate of hospital admissions, ICU care, need of IMV, and mortality between a control group of 330 patients with obesity and 33 patients with a prior bariatric surgery who tested positive for COVID-19. Patients with prior bariatric surgery had a lower rate of hospitalization, no need for ICU or IMV, and no mortality [31]. These results were further confirmed by a recently published meta-analysis that included patients with prior bariatric surgery and COVID-19 infection, which showed $78 \%$ and $72 \%$ risk reductions in mortality and hospitalization compared to patients with obesity without bariatric surgery [16]. Altogether, these findings indicate that bariatric surgery appears to protect a high-risk population from severe COVID-19 infection in the current pandemic context. 
Our study has limitations and strengths. One limitation is that the testing for postoperative COVID-19 infection was only performed in symptomatic patients, which could have underestimated the overall rate of postoperative COVID-19 infection. However, asymptomatic COVID-19 infection poses no significant risk for severe complications, and thus the clinical impact is limited. Also, it is important to consider that the opening phase for bariatric surgery occurred when SARS-CoV-2 viral circulation levels were low; thus, we cannot extrapolate these results when circulating viral levels are higher. A strength of our study is that we have a $100 \%$ follow-up of all our 189 patients during the first 30 days after discharge, and we were able to use the same rigorous COVID-19 protocol and recommendations that we used in the early phases of the pandemic for unpostponable surgeries, with positive results [4].

The actual COVID-19 pandemic is a serious risk for patients with obesity, as shown by higher rates of severe infection, hospitalization, and mortality. While confinement measures have an emphasis on decreasing the spread of the virus, protecting vulnerable populations which include patients with obesity, at the same time these have limited the comprehensive care of this group of patients. Evidence has shown that confinement promotes unhealthy diets, decreases physical activity, affects mental health, and delays medical care [32], in addition to the deleterious effects of restriction and limitation to bariatric surgery [33]. Therefore, the safe resumption of bariatric surgery during this pandemic is of critical importance.

Our results summarize the clinical outcomes of consecutive patients who underwent primary bariatric surgery between the first and second waves of the COVID-19 pandemic in Chile. New studies and other experimental designs will be necessary to determine the safety of bariatric surgery procedures during this pandemic and elucidate its role with COVID-19 infection and its effectiveness regarding short- and long-term weight loss.

\section{Conclusion}

Bariatric surgery can still be safely performed during the current pandemic with a low risk of postoperative symptomatic COVID-19 and severe complications. Due to the safety profile of bariatric surgery and the increased risk of severe complications due to COVID-19 infection, resumption of surgical treatment of obesity and its associated diseases must be based on local criteria and adjusted protocols from current guidelines to offer a safe procedure.

\section{Declarations}

Ethics Approval For this type of study, formal consent is not required.

Informed Consent Informed consent was obtained from all individual participants included in the study.

Conflict of Interest The authors declare no competing interests.

\section{References}

1. Collaborative C. Mortality and pulmonary complications in patients undergoing surgery with perioperative SARSCoV-2 infection: an international cohort study. Lancet. 2020;396(10243):27-38. https://doi.org/10.1016/S0140-6736(20) 31182-X.

2. Aminian A, Safari S, Razeghian-Jahromi A, Ghorbani M, Delaney CP. COVID-19 Outbreak and surgical practice: unexpected fatality in perioperative period. Ann Surg. 2020;272(1):e27-9. https:// doi.org/10.1097/SLA.0000000000003925.

3. Aminian A, Kermansaravi M, Azizi S, Alibeigi P, Safamanesh S, Mousavimaleki A, et al. Bariatric surgical practice during the initial phase of COVID-19 outbreak. Obes Surg. 2020;30(9):3624-7. https://doi.org/10.1007/s11695-020-04617-x.

4. Inzunza M, Romero C, Irarrazaval MJ, Ruiz-Esquide M, Achurra P, Quezada N, et al. Morbidity and mortality in patients with perioperative COVID-19 infection: prospective cohort in general, gastroesophagic, hepatobiliary, and colorectal surgery. World J Surg. 2021;45(6):1652-62. https://doi.org/10.1007/ s00268-021-06068-6.

5. Zhang X, Lewis AM, Moley JR, Brestoff JR. A systematic review and meta-analysis of obesity and COVID-19 outcomes. Sci Rep. 2021;11(1):7193. https://doi.org/10.1038/s41598-021-86694-1.

6. Stefan N, Birkenfeld AL, Schulze MB, Ludwig DS. Obesity and impaired metabolic health in patients with COVID-19. Nat Rev Endocrinol. 2020;16(7):341-2. https://doi.org/10.1038/ s41574-020-0364-6.

7. Zhou Y, Chi J, Lv W, Wang Y. Obesity and diabetes as high-risk factors for severe coronavirus disease 2019 (Covid-19). Diabetes Metab Res Rev. 2021;37(2):e3377. https://doi.org/10.1002/dmrr. 3377.

8. Popkin BM, Du S, Green WD, Beck MA, Algaith T, Herbst CH, et al. Individuals with obesity and COVID-19: a global perspective on the epidemiology and biological relationships. Obes Rev. 2020;21(11):e13128. https://doi.org/10.1111/obr.13128.

9. Hamer M, Gale CR, Kivimaki M, Batty GD. Overweight, obesity, and risk of hospitalization for COVID-19: a community-based cohort study of adults in the United Kingdom. Proc Natl Acad Sci U S A. 2020;117(35):21011-3. https://doi.org/10.1073/pnas. 2011086117.

10. Rubino F, Cohen RV, Mingrone G, le Roux CW, Mechanick JI, Arterburn DE, et al. Bariatric and metabolic surgery during and after the COVID-19 pandemic: DSS recommendations for management of surgical candidates and postoperative patients and prioritisation of access to surgery. Lancet Diabetes Endocrinol. 2020;8(7):640-8. https://doi.org/10.1016/S2213-8587(20) 30157-1.

11. Yang W, Wang C, Shikora S, Kow L. Recommendations for metabolic and bariatric surgery during the COVID-19 pandemic from IFSO. Obes Surg. 2020;30(6):2071-3. https://doi.org/10.1007/ s11695-020-04578-1.

12. Behrens E, Poggi L, Aparicio S, Martínez Duartez $\mathrm{P}$, Rodríguez N, Zundel N, et al. COVID-19: IFSO LAC 
Recommendations for the resumption of elective bariatric surgery. Obes Surg. 2020;30(11):4519-28. https://doi.org/10.1007/ s11695-020-04910-9.

13. Singhal R, Tahrani AA, Ludwig C, Mahawar K, collaborators G. Global 30-day outcomes after bariatric surgery during the COVID-19 pandemic (GENEVA): an international cohort study. Lancet Diabetes Endocrinol. 2021;9(1):7-9. https://doi.org/10. 1016/S2213-8587(20)30375-2.

14. Yerdel MA, Ozgen G. Eighty-seven consecutive sleeve gastrectomies between the two peaks of the covid-19 pandemic. An opening-phase experience Obes Surg. 2021;31(7):3026-30. https://doi. org/10.1007/s11695-021-05339-4.

15. Balieiro MPM, da Silva MM, Coelho ACJ, de Barros F, Saboya C, Ferraz LR, et al. Restarting elective bariatric and metabolic surgery under a security protocol during the COVID-19 pandemic-a prospective observational cohort study. Obes Surg. 2021;31(7):3083-9. https://doi.org/10.1007/s11695-021-05368-z.

16. Aminian A, Tu C. Association of bariatric surgery with clinical outcomes of SARS-CoV-2 infection: a systematic review and meta-analysis in the initial phase of COVID-19 pandemic. Obes Surg. 2021;31(6):2419-25. https://doi.org/10.1007/ s11695-020-05213-9.

17. Dindo D, Demartines N, Clavien PA. Classification of surgical complications: a new proposal with evaluation in a cohort of 6336 patients and results of a survey. Ann Surg. 2004;240(2):205-13.

18. Brethauer SA, Kim J, El Chaar M, Papasavas P, Eisenberg D, Rogers A, et al. Standardized outcomes reporting in metabolic and bariatric surgery. Obes Surg. 2015;25(4):587-606. https:// doi.org/10.1007/s11695-015-1645-3.

19. Irarrazaval MJ, Inzunza M, Munoz R, Quezada N, Branes A, Gabrielli M, et al. Telemedicine for postoperative follow-up, virtual surgical clinics during COVID-19 pandemic. Surg Endosc. 2020. https://doi.org/10.1007/s00464-020-08130-1.

20. Romero-Velez G, Pereira X, Skendelas JP, Costinett S, Grosser R, Creange C, et al. Diagnosis of COVID-19 and the bariatric surgery population: a single center experience. Surg Endosc. 2021. https://doi.org/10.1007/s00464-020-08249-1.

21. Lei S, Jiang F, Su W, Chen C, Chen J, Mei W, et al. Clinical characteristics and outcomes of patients undergoing surgeries during the incubation period of COVID-19 infection. EClinicalMedicine. 2020:100331. https://doi.org/10.1016/j.eclinm.2020.100331

22. Luong-Nguyen M, Hermand H, Abdalla S, Cabrit N, Hobeika C, Brouquet A, et al. Nosocomial infection with SARS-Cov-2 within departments of digestive surgery. J Visc Surg. 2020;157(3S1):S13S8. https://doi.org/10.1016/j.jviscsurg.2020.04.016

23. Doglietto F, Vezzoli M, Gheza F, Lussardi GL, Domenicucci M, Vecchiarelli L, et al. Factors associated with surgical mortality and complications among patients with and without coronavirus disease 2019 (COVID-19) in Italy. JAMA Surg. 2020. https://doi. org/10.1001/jamasurg.2020.2713

24. Cai M, Wang G, Zhang L, Gao J, Xia Z, Zhang P, et al. Performing abdominal surgery during the COVID-19 epidemic in Wuhan, China: a single-centred, retrospective, observational study. Br J Surg. 2020;107(7):e183-5. https://doi.org/10.1002/bjs.11643.
25. Fu D, Zhang P, Wang L, Liu W, Tan H, Di M, et al. Emergency abdominal surgery in COVID-19 patients: a note of caution from Wuhan. Br J Surg. 2020;107(8):e262. https://doi.org/10.1002/bjs. 11722.

26. McDermott A, O'Kelly J, de Barra E, Fitzpatrick F, Little DM, Davis NF. Perioperative outcomes of urological surgery in patients with SARS-CoV-2 infection. Eur Urol. 2020;78(1):11820. https://doi.org/10.1016/j.eururo.2020.05.012.

27. Di Martino MS, JavierMaqueda González, RocíoMuñoz de Nova, Jose Luisde la Hoz Rodríguez, ÁngelaCorrea Bonito, AlbaMartínPérez, Elena. Cirugía electiva durante la pandemia por SARSCoV-2 (COVID-19): análisis de morbimortalidad y recomendaciones sobre priorización de los pacientes y medidas de seguridad. Cirugía Española. 2020. https://doi.org/10.1016/j.ciresp.2020.04. 029.

28. Álvarez Gallego MGdlC, SaraPascual Migueláñez, IsabelRubioPérez, InésBarragán Serrano, CristinaÁlvarez Peña, EstíbalizDíaz Domínguez, Joaquín. Impacto de la pandemia por SARS-CoV-2 sobre la actividad y profesionales de un Servicio de Cirugía General y del Aparato Digestivo en un hospital terciario. Cirugia Española. 2020;98(6):320-7. https://doi.org/10.1016/j.ciresp. 2020.04.001.

29. Pérez-Rubio Á, Sebastián Tomás JC, Navarro-Martínez S, Gonzálvez Guardiola P, Torrecillas Meroño DG, Domingo Del Pozo C. Incidence of surgical abdominal emergencies during SARS-CoV-2 pandemic. Cir Esp. 2020. https://doi.org/10.1016/j. ciresp.2020.06.017.

30. Aminian A, Brethauer SA, Kirwan JP, Kashyap SR, Burguera B, Schauer PR. How safe is metabolic/diabetes surgery? Diabetes Obes Metab. 2015;17(2):198-201. https://doi.org/10.1111/dom. 12405 .

31. Aminian A, Fathalizadeh A, Tu C, Butsch WS, Pantalone KM, Griebeler ML, et al. Association of prior metabolic and bariatric surgery with severity of coronavirus disease 2019 (COVID-19) in patients with obesity. Surg Obes Relat Dis. 2021;17(1):208-14. https://doi.org/10.1016/j.soard.2020.10.026.

32. Beran D, Aebischer Perone S, Castellsague Perolini M, Chappuis F, Chopard P, Haller DM, et al. Beyond the virus: ensuring continuity of care for people with diabetes during COVID-19. Prim Care Diabetes. 2021;15(1):16-7. https://doi.org/10.1016/j. pcd.2020.05.014.

33. Ahmed B, Altarawni M, Ellison J, Alkhaffaf BH. Serious impacts of postponing bariatric surgery as a result of the COVID-19 pandemic: the patient perspective. J Patient Exp. 2021;8:23743735211008280. https://doi.org/10.1177/2374373521 1008282 .

Publisher's Note Springer Nature remains neutral with regard to jurisdictional claims in published maps and institutional affiliations. 